\title{
Using IMS Learning Design to Author Authentic Learning Examples in Mobile Context
}

\author{
Ryan Jesse, Kinshuk, Maiga Chang \\ School of Computing and Information Systems \\ Athabasca University \\ Athabasca, Canada \\ ryan.jesse@usask.ca,kinshuk@athabascau.ca,maigac@athabascau.ca
}

\begin{abstract}
Modern mobile devices are equipped with input and sensor options that allow for user generated content, which can be used to create examples of real life learning situations, or authentic learning examples. However, existing research shows a gap between the creation of authentic learning examples and their subsequent reuse as learning objects. Therefore, the goal of this research is to design a mechanism to author authentic learning examples in a standardized format to enable reuse using mobile devices. The implementation, Mobile Authentic Authoring in IMS (MAAIMS), captures authentic learning examples with the mobile device sensors (still camera, video camera, microphone) which can be supplemented with location-aware GPS coordinates and other descriptive metadata following IMS Metadata specifications. MAAIMS encapsulates these authentic learning examples and employs them as standardized learning objects (IMS Content Packages), and optionally as, standardized learning activities (IMS Learning Designs). The mechanism allows for sharing and reuse of authentically created learning examples and learning activities in multiple contexts.
\end{abstract}

Mobile learning; IMS Learning Design; instructional design; learning object; authentic learning, metadata, mobile sensor

\section{INTRODUCTION}

E-learning technologies have allowed authoring and playback of standardized reusable learning objects (RLOs) for several years. Effective mobile learning requires similar functionality at both design time and runtime. Mobile devices can play RLOs using applications like SMILE, mobile access to an LMS, or other systems which deploy content to mobile learners [1-3]. However, implementations which author content in a mobile context do not typically permit reuse across multiple contexts due to a lack of standardization. Standards based (IMS and SCORM) authoring implementations exist for non-mobile platforms [4-6]. However, this paradigm precludes capturing learning where and when it occurs. Consequently, RLOs authored for e-learning lack social constructivism pedagogies for learner generated content, especially with timely, relevant, and location aware examples.
Nowadays, mobile devices are equipped with input and sensor options that allow user-generated content, which may be used to create examples of authentic learning. An implementation of a system using mobile devices for creation of authentic examples is presented in [7]. However, this system does not implement standardization or a method for exporting content beyond the system in which it was created. Herein lies the traditional boundary to reuse; standardization of content is required explore reuse in a multitude of other tools implementing the same standards.

The RAFT project is capable of capturing images and audio in a mobile content, and tagging with metadata [8]. However, content reuse within a learning design or standardized content is not explored. Other implementations that allow for mobile capture and publishing of learning content do not typically reuse the content beyond the system in which it was authored, nor is the capture process integrated [9-10].

Several projects address location aware adaptive mobile learning [11-12]. Thus, it is critical that this project includes this contextual metadata when authoring learning content so it extends the possibility to be used as a basis for adaptivity at runtime.

Given this status of research, our research questions are as follows:

1. How can mobile device sensor data be used to author authentic learning examples for use in reusable learning objects?

2. How can these learning objects be utilized in context to create learning designs conforming to IMS standards for sharing across contextual boundaries?

These research questions are reflected in the conceptual design (Figure 1) of the tool. Learners could use the tool to capture learning scenarios and situations from their daily life, where and when they occur. These authentic learning situations are captured as authentic learning examples, and can be shared with other co-learners across multiple systems and contexts, like learning management systems. Moreover, activities can be created in the tool to utilize the authentic learning examples via a learning design unit of learning playable in a runtime environment. The tool can be used by instructional designers to develop inquiry based course content or by students for sharing with co-learners in social constructivism, collaborative leraning, or as the basis for assessment. 


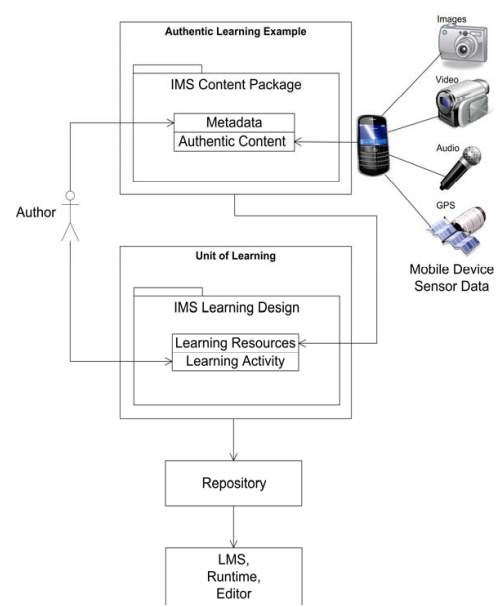

Figure 1. Conceptual design of the mobile authentic authoring tool.

\section{SYSTEM ARCHITECTURE}

\section{A. Proposed Solutions}

In order to achieve the task of creating IMS standard learning objects in a mobile context, a variety of solutions have been investigated.

IMS Global Learning Consortium Metadata, Content Packaging, and Learning Design specifications were selected as they are well defined and widely implemented within elearning. Together, these standards permit the creation of metadata, reusable learning objects, and learning activities within a single, portable package.

A web site optimized for use on a mobile device browser was explored which would permit cross platform usage. However, this solution was deemed less feasible because experimentation determined that a web page would have compatibility issues interfacing with sensors, such as the embedded camera, across a wide variety of mobile hardware, mobile browsers and mobile operating systems. A standalone application was also investigated. Since the learning design would need to be written on the mobile device, this approach may hinder sharing of the resulting learning resources as the content would only exist on the mobile device. A discrete task would be required to upload the learning content to a repository for sharing with others.

As a result, the implementation solution is a two-tiered application consisting of a mobile client and a server component.

\section{B. Mobile Component}

The resulting solution is a mobile application, entitled "MAAIMS", which is an acronym for Mobile Authentic Authoring in IMS. The MAAIMS client runs on smart phone, specifically, Research in Motion devices with Blackberry OS 5, 6 or 7 installed.

The component running on the mobile device is a compiled and installed executable application which utilizes web standards like HTML5, CSS, JavaScript, and AJAX running inside the WebWorks Platform hosted on the operating system. This component presents the user interface and interacts with the IO channels and mobile device sensors, such as the touch screen, keyboard, the global positioning sensor, the embedded camera, and the microphone.

The mobile client connects to a server for uploading metadata and authentic learning content, and subsequently for downloading the resulting content package to local storage on the mobile device. By default, this ensures that learning content is stored on the server, which acts as a repository. However, it necessitates that the mobile device is periodically connected to the internet during application execution. Given the rapid proliferation of $3 \mathrm{G}$ and $4 \mathrm{G}$ mobile data networks, along with Wi-Fi coverage in many educational institutes, the connectivity requirement of this application was deemed an acceptable requirement.

The WebWorks SDK provides access to the BlackBerry ecosystem via the API for interfacing with native applications such as the audio recorder, video recorder, camera and media player.

Where possible, the third party PhoneGap API was used. This framework allows a common API across multiple mobile platforms, like BlackBerry, Android, and iPhone, which permits easier porting when compared to a native application solution. Additionally, the WebKit browser engine implements the WC3 Geolocation API, which allows a standardized interface to query the geographical location in a GPS tuple of longitude and latitude which are utilized to individually tag authentic learning examples with their capture location.

MAAIMS is designed to capture real-life learning examples, such as fieldwork demonstrations. Thus, outdoor examples which are location dependant can be geo-tagged. Learners could thereby visit a location of a learning example, or location data could enable adaptive location based learning. For example, artifacts during a field trip could be captured and activities could be authored to make use of the learning derived from the authentic learning examples such as exercises, problems, or questions. The resulting standardized output can be shared in an e-learning, mobile learning, or blended learning environment.

\section{Server Component}

The server interfaces with the mobile client over standard HTTP and TCP/IP. The server component accepts the metadata and multimedia uploaded by the client, stores it in a database, and then dynamically generates HTML (environments, activities, objective, prerequisite, and resources) and XML (imsmanifest.xml) according to the IMS standards, and bundles generated content and multimedia into a package interchange file. This component resides on an internet connected Linux machine hosting Apache, MySQL and PHP. The generated XML and HTML is written by DOM. Imsmanifest.xml is valid against several XML schemata: IMS Content Package schema, IMS Metadata schema and, optionally, IMS Learning Design schema.

The package interchange file is a uniquely named .zip file containing all of the learning resource HTML files, the imsmanifest.xml, and the captured multimedia files 
representing authentic learning objects. The zip file is stored on the server for the mobile client to download to local storage and a copy is retained on the server in the web-based repository for reuse.

\section{MethODOLOGY}

This methodology subscribes to the design for learning design authoring tools as developed by [13] wherein course materials should be developed as a set of learning objects as the first step in an IMS LD authoring tool. Secondly, metadata should be added to those learning objects. Finally, the published methodology states that instructional design should be added.

\section{A. Collect Learning Object Metadata}

The metadata fields collected follow the IMS Metadata specifications based on the IEEE/LOM Metadata. Figure 2(a) shows the initial screenshot of the mobile component where IMS Metadata is collected which represents the general and lifecycle elements and describes the learning object as a whole. The technical section of the metadata in the imsmanifest.xml is entirely application generated. This section describes the technical contents of the resources in the content package, including requirements to view the content at runtime, file types included in the package, and the location at which the content package is available. Wherever possible, metadata is application generated to minimize user input. However, some fields are set as required fields because additional metadata availability means increased searchability and reusability.

In Figure 2(b), the educational and pedagogical metadata is presented after the collection of authentic learning examples, because the responses to fields such as typical learning time will depend on the content, number, and type of learning examples collected.

\section{B. Collect Authentic Learning Examples}

Key to this project is the use of mobile sensors to capture authentic learning examples. The MAAIMS application achieves this task by integrating with the native applications of the mobile device for three mediums of media capture. GPS location and learning example description are also independently obtained for each example.
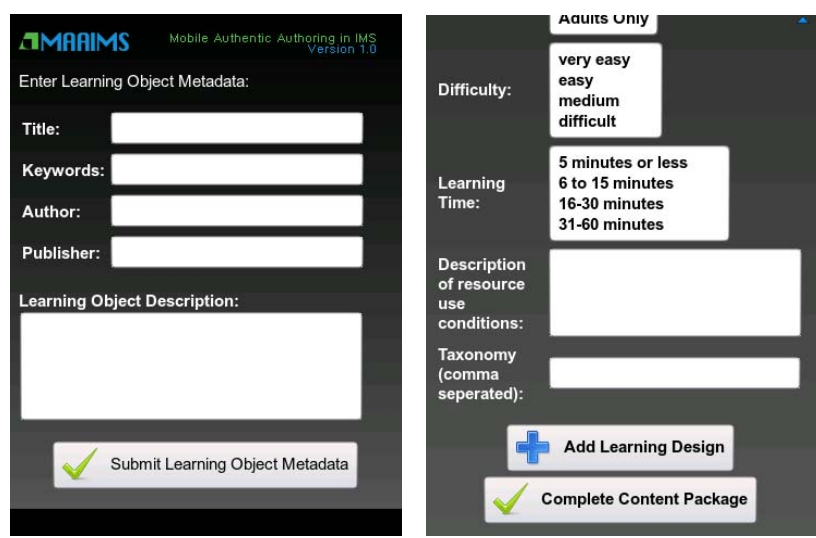

Figure 2. RLO metadata collection in MAAIMS. (a) represents general metadata and (b) collects educational metadata.
After the initial learning object metadata has been submitted, the application presents options for each type of authentic learning example capture. If the user selects "Capture Audio," "Capture Picture," or "Capture Video," the application will launch the respective recording application (audio recorder, camera, video camera). The user can then capture the authentic learning example by recording a learning scenario where and when it occurs. The GPS coordinates are queried simultaneously as

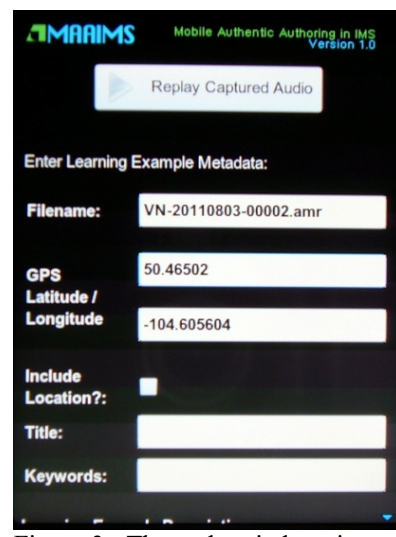

Figure 3. The authentic learning example capture process shows the captured filename, location, and example description. each authentic learning example is captured.

Each authentic learning example can be replayed, discarded and recaptured from within the MAAIMS application to ensure sufficient quality or educational value was captured before tagging with metadata, or including in the content package as seen in Figure 3.

Once a suitable capture has been completed and the required metadata has been entered, the user can press submit the metadata and multimedia. This uploads the media to binary file storage on the web server, and the corresponding example metadata to the database. The capture process can be repeated multiple times, with any combination of media types.

An important consideration is the selected capture resolution of the media which affects the file size and upload time. However, the lower the quality, the more limited the possible contexts for reuse become.

\section{Complete Content Package without Learning Design}

Once the completed capture option has been pressed, the application collects educational metadata as previously discussed in methodology part A. Prior to submitting the educational metadata, the user is presented with two options, as seen in Figure 2(b). "Add Learning Design" will create learning activity within the content package, and "Complete Content Package" will complete the content package without embedded learning design. If the user opts to complete the content package, the server generates the physical files (actual media representing authentic learning objects), and the manifest (containing the metadata, resources, and organizations sections), compiles into a standalone package, and shares to the repository. Before exiting, the mobile user can save the content package to the mobile device. This is useful if importing to a system which only supports IMS Content Packaging and not IMS Learning Design or for sharing content without utilizing activities.

\section{Add Optional Learning Design}

This step displays how to create a content package including IMS Learning Design Level A, accessible by the 


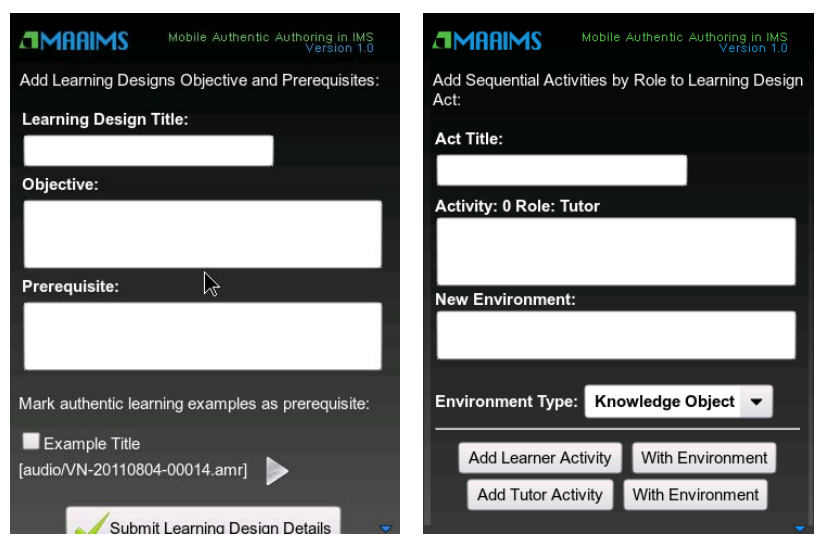

Figure 4. Learning Design objectives and prerequisites are created or authentic learning examples can be tagged as prerequisites, and (b) displays activities and environment creation within an act.

"Add Learning Design" button in Figure 2(b). The IMS Learning Design information model specifies title, learningobjectives, and prerequisites as learning design elements, which are collected in Figure 4(a).

The learning objective field represents the overall goals to be met by learners who complete the activities in the learning design and the authentic learning examples contained in the content package. Next, Figure 4(a) requests the user input prerequisites. Previously captured authentic learning objects can be tagged as a prerequisite to the unit of learning. The check box representing each of the previously captured learning examples can be selected if the example specifies one of the entry requirements for interacting with the learning design. Figure 4(a) only displays one authentic learning example, but all examples captured in the same instance will be listed for referencing as prerequisites.

Activities can be added to an act. Pressing "Add Learner Activity" or "Add Tutor Activity" will create a new activity, assigned to the respective role, in the current act, as shown in Figure 4(b). Related activities are created within the same act. Activities can also be created with an environment that creates a relationship between the activity and an environment within which the activity is executed. The environment could represent a learning object, service, or tool that is available to the learner at runtime. The environment type is set by the author as seen in the drop down selection of Figure 4(b).

Previously created authentic learning examples can be referenced within an act in a similar manner to how they are referenced as a prerequisite, as shown in Figure 4(a). These LD references reuse content package resources. If an authentic learning example is referenced within an act, it would be considered a relevant learning object to the activities contained within the act and would be treated as reference material for completing the activities defined.

The add additional act option will insert another act into the learning design, and will repeat the process of adding activities, environments, and referencing authentic learning examples until the defined learning objective can be met.

Once the learning design has been completed, the package interchange file, including learning design, is generated by the server which can be accessed from the mobile client or the repository.

This methodology demonstrates that mobile device sensor data can be utilized to author authentic learning examples. These authentic learning examples are, in turn, utilized within IMS Content Packages and IMS Learning Designs. The MAAIMS implementation indicates that a mobile application can complete these tasks in a mobile context.

\section{FINDINGS}

The remaining research goal is to share the created content packages in other contexts beyond the mobile platform in which they were authored. This goal seeks to demonstrate the reusability and applications of MAAIMS output.

\section{A. Repository}

Repositories are collections of learning objects which can be stored, indexed, and retrieved for reuse; thus a repository is required to address the sharing and reuse research goal. A repository is an intermediary between the MAAIMS authoring environment and the context of reuse.

Each package interchange file created by MAAIMS is listed in the web-based MAAIMS repository. By clicking the unique identifier of the package interchange file, the entire content package can be saved locally.

\section{B. Validation}

To confirm that the learning design produced by MAAIMS is compliant to the standards, CopperCore Version 3.3 is used to validate the package interchange file. A .zip file, from the MAAIMS repository, or stored locally on the mobile device can be input into CopperCore for validation. The validation engine checks the XML compliance against the schema, ensures that the content is properly referenced, and that the learning design is semantically correct. A successful validation of the learning design supports the assertion that MAAIMS can produce valid IMS Learning Designs in a mobile context.

\section{Learning Management Systems}

Key to reuse of MAAIMS created content is import into a learning management system (LMS) that can deliver webbased learning for distance education, continuing education, or blended classrooms. Thus authentic learning examples can to be imported and shared with learners using an LMS.

Moodle 1.9 and 2.0 do not implement IMS Learning Design support. As a result, only IMS Content Packages that do not include learning design could be tested using Moodle. The package interchange files were successfully loaded and shared with learners. A hyperlink to the multimedia authentic learning example is provided in each resource. Clicking this link will open the image, audio, or video. Clicking the map location link will display a map of where the authentic learning example was captured assuming the author opted to include the location in the metadata.

It remains important to import IMS Learning Design in an LMS (dotLRN) to demonstrate MAAIMS content in an e- 
learning context. The package interchange file can successfully be imported from the repository and dotLRN LMS users can be assigned to the roles in the LD. A new instance of the learning design runtime can then be created and executed, showing that all MAAIMS created IMS Learning Design elements, such as activities, roles, environments, authentic learning examples as resources, prerequisites, and objectives are successfully processed at runtime in an LMS.

\section{IMS Authoring and Editing Tools}

The Reload Project serves to "provide the crucial 'missing link' which allows users to author and transfer learning objects, in specification compliant format, between authoring and design tools... and VLEs [14]." Reload was successfully used to open, edit, and augment IMS Content Packages and IMS Learning Design MAAIMS resources.

\section{E. Runtime Environment}

Learning design must be able to be played, executed, or run using a runtime environment. These runtime platforms consist of a server that can read the IMS Learning Design unit of learning, and coordinate the activities carried out between roles. Runtime systems tested included CopperCore Learning Design Player, Reload Learning Design Player, Service-based Learning Design (SLeD), Astro Learning Design Player. All elements and components created in MAAIMS were executed successfully and all runtime interactions were as expected which demonstrates cross context reuse of content, metadata and activities captured in a mobile context in a runtime environment.

\section{CONCLUSION}

\section{A. Future Work}

An empirical study will be conducted with a group of grade 9 students who will have access to Android based mobile devices. As such, an Android version of MAAIMS will be developed to research the learner applications, usage scenarios, and effectiveness.

Initial testing was completed with Moodle, in a mobile context, using MAAIMS content packages without LD. This allows learning object authoring and playback to be completed in a mobile context; conceptually completing the mobile learning cycle wherein learning content authoring (design time) and playback (runtime) occur in a mobile context. However, further testing is required with SMILE PDA Learning Design Player as a mobile runtime environment utilizing MAAIMS-generated LD content.

\section{B. Conclusion}

This paper presents MAAIMS, an implemented mobile authoring tool for standardized learning content. MAAIMS novelty stems from the smooth transition and integration between metadata collection, authentic learning example collection, and learning design authoring. The tool demonstrates authoring authentic learning examples with location-awareness in a mobile context, and utilization of the authentic learning examples in IMS Metadata, IMS Content Package and IMS Learning Design. Content is shareable via the repository, is validated against the standards, and reusable in multiple contexts such as editing tools, runtime environments, and learning management systems. Thus, the research questions have been conclusively answered.

\section{ACKNOWLEDGMENT}

Authors acknowledge the support of NSERC, iCORE, Xerox, and the research related funding by Mr. A. Markin.

\section{REFERENCES}

[1] S. Castillo and G. Ayala, "ARMOLEO: an architecture for mobile learning objects," in Electronics, Communications and Computers, 2008. CONIELECOMP 2008, 18th International Conference on, Washington, DC, 2008, pp. 53-58.

[2] T. Y. Hsu and C. M. Chen, "A Mobile Learning Module for High School Fieldwork.," Journal of Geography, vol. 109, no. 4, p. 9, 2010.

[3] I. A. Zualkernan, S. Nikkhah, and M. Al-Sabah, "A Lightweight Distributed Implementation of IMS LD on Google's Android Platform," in 2009 Ninth IEEE International Conference on Advanced Learning Technologies, 2009, pp. 59-63.

[4] V. Gonzalez-Barbone and L. Anido-Rifon, "Creating the first SCORM object," Computers \& Education, vol. 51, no. 4, pp. 1634$1647,2008$.

[5] D. Griffiths, P. Beauvoir, O. Liber, and M. Barrett-Baxendale, "From reload to ReCourse: learning from IMS learning design implementations," Distance Education, vol. 30, no. 2, pp. 201-222, 2009.

[6] A. G. Téllez, "Authoring environment for e-learning production based on independent XML formats," in Education Engineering (EDUCON), 2010 IEEE, 2010, pp. 415-422.

[7] Y. H. Kuo, Y. M. Huang, T. C. Liu, and M. Chang, "Collaborative Creation of Authentic Examples with Location for u-Learning," Proc. e-Learning, vol. 2, pp. 16-20, 2008.

[8] M. Specht and M. Kravcik, "Authoring of learning objects in context," International Journal on E-Learning, vol. 5, no. 1, p. 25, 2006.

[9] K. Kiili, J. Multisilta, M. Suominen, and H. Ketamo, "Learning experiences of mobile social media," International Journal of Mobile Learning and Organisation, vol. 4, no. 4, pp. 346-359, 2010.

[10] A. Comas-Quinn, R. Mardomingo, and C. Valentine, "Mobile blogs in language learning: making the most of informal and situated learning opportunities," ReCALL, vol. 21, no. 1, pp. 96-112, 2009.

[11] J. Y. K. Yau and M. Joy, "A context-aware personalised m-learning application based on m-learning preferences," International Journal of Mobile Learning and Organisation, vol. 5, no. 1, pp. 114, 2011.

[12] M. Svensson, "Contextual metadata in practice," in Advances in Multimedia, 2009. MMEDIA'09. First International Conference on, Colmar, France, 2009, pp. 12-17.

[13] J. G. Boticario and O. C. Santos, "An open IMS-based user modelling approach for developing adaptive learning management systems," Journal of Interactive Media in Education, vol. 2, 2007.

[14] P. Beauvoir, "RELOAD Project," 05-2011. [Online]. Available: http://www.reload.ac.uk/. [Accessed: 11-Sep-2011]. 